\title{
The Fortran Parallel Transformer and its Programming Environment
}

\author{
Erik H. D'Hollander, Fubo Zhang and Qi Wang \\ University of Ghent \\ Department of Electrical Engineering \\ Parallel Information Systems group \\ St.-Pietersnieuwstraat 41 \\ B-9000 Gent, Belgium \\ email: $\quad\{$ dhollander, wang $\} @ e l i s . r u g . a c . b e$ \\ zfb@platform.com \\ tel: $\quad+32-9-264.33 .75$ \\ keywords: programming environment, program restructuring, PVM, \\ Fortran parallelizer, automatic code generation \\ DOALL, distributed computing
}

\begin{abstract}
The Fortran Parallel Transformer (FPT) is a Parallel Programming Environment for Fortran-77 programs. It is used for the automatic parallelization of loops, program transformations, dependence analysis, performance tuning and code generation for various platforms. FPT is able to deal with GOTO's by restructuring ill-structured code using hammock graph transformations. In this way more parallelism becomes detectable.

The X-window based Programming Environment, PEFPT, extends FPT with interactive dependence analysis, the iteration space graph and guided loop optimization.

FPT contains a PVM (Parallel Virtual Machine) code generator which converts the parallel loops into PVM master- and slave-code for a network of workstations. This includes job scheduling, synchronization and optimized data communication. The productivity gained is about a factor of ten in programming time and a significant speedup of the execution.
\end{abstract}




\section{Introduction}

The Fortran Parallel Transformer is a compilation system which analyzes serial source code and finds parallel loops. Output is generated for shared memory multiprocessors and message passing distributed computers. In addition, PEFPT, a Programming Environment for FPT, is a X-Window based parallel program development tool which assists the user with graphical views of the program. The X-Window programming system is presented in section 2. The highlights of the FPT environment are the user assisted dependence analysis based on the iteration space graph, the code restructuring and the generation of distributed PVM-code.

FPT recognizes parallel loop iterations using well known dependence analysis techniques[8]. Sometimes however, the analytical techniques fail to discover even obvious parallel iterations. In that case, the iteration space graph (ISG) is used to depict the dependences by type and by variable. The ISG shows how iterations interfere and this allows users to safely parallelize code which is otherwise intractable by analytical tests. The dependence analysis and the use of the iteration space graph and are presented in section 3 .

Code restructuring is concerned with repairing the ill-structured control flow caused by non-blocked branches. The presence of goto's or if-goto's obscures the execution trajectory and prevents the detection of the parallelism. Using hammock graph transformations, the so-called spaghetti code is transformed into well-structured code blocks, amenable for parallel execution. This is discussed in section 4.

Distributed computing provides high performance computing by assigning relatively independent components of a program to a cluster of computers and executing them in parallel. The Parallel Virtual Machine (PVM) concept is a powerful programming model to distribute a parallel application on a network of cooperative computers. In section 5 the techniques employed by FPT to discover and amplify the parallelism in a program are extended with a code and message passing generator for PVM. An algorithm to minimize data communication is presented and example programs are given. In section 6 the techniques used in FPT to detect and exploit the parallelism are illustrated for a number of typical programs, such as the Jacobi and Fast Fourier Transform and a PVM benchmark. In section 7 the concluding remarks and future directions of this work are given. 


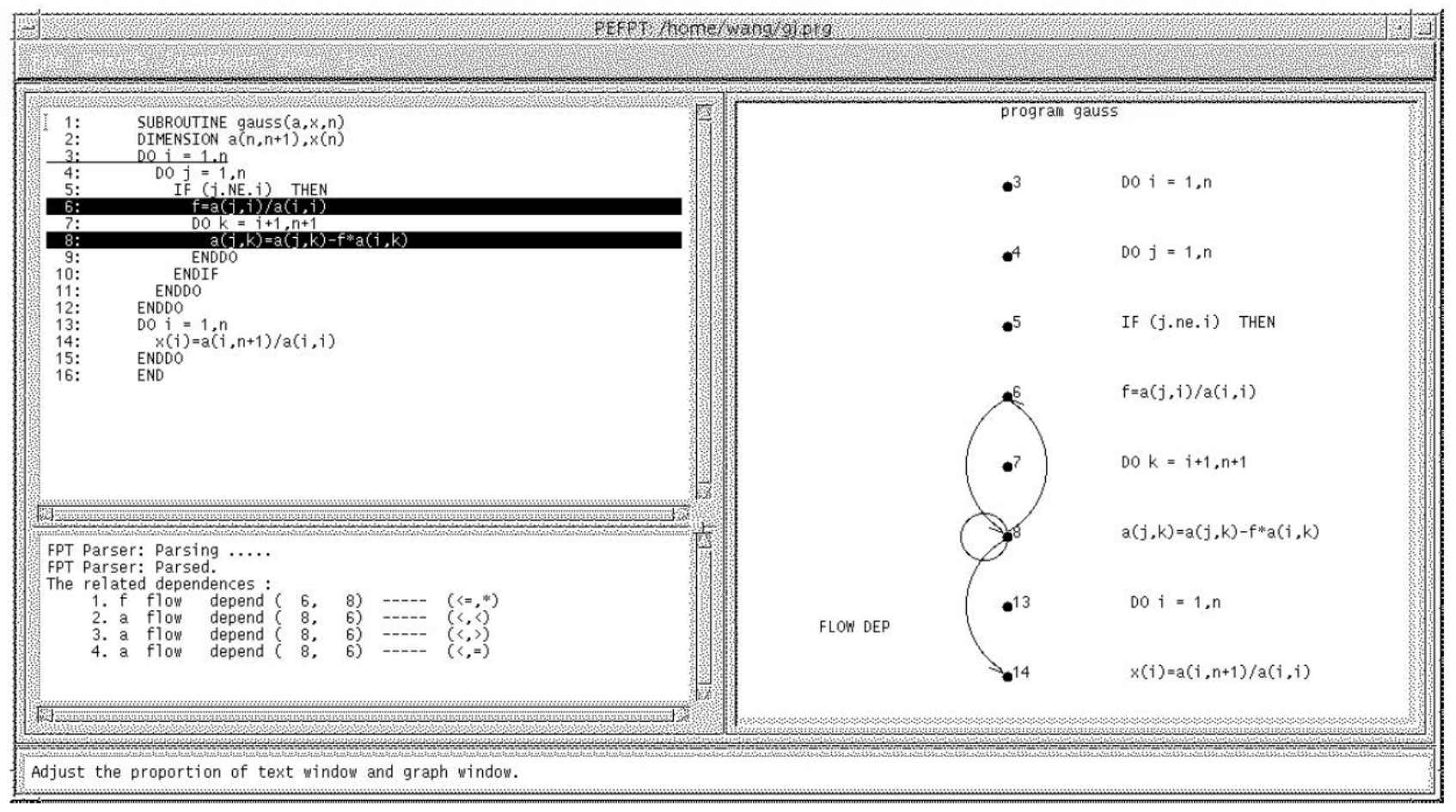

Figure 1: The opening canvas of the FPT Programming Environment contains a program window, a graphical window for visual program analysis and the textual output window. When clicking a dependence arc in the graphic window, the corresponding statements are highlighted.

\section{The FPT parallel programming environment}

FPT is a $>40.000$ lines toolkit for the parallelization of $\mathrm{f} 77$ programs. In its noninteractive form, FPT detects parallel loops using well-known dependence analysis techniques[8], and generates a number of output files:

$$
\begin{array}{ll}
\cdot \text { prg } & =\text { generated code (multithreaded, PVM, Fortran/MP...) } \\
\text {.tsk } & =\text { annotated assembly code } \\
\text { why } & =\text { explains dependences preventing parallel execution } \\
\text { call.ps } & =\text { call graph } \\
\text { dep.ps } & =\text { dependence graph }
\end{array}
$$

In its interactive form, an $\mathrm{X}$ canvas is offered with three panes: the program, the output and the graphical window (see figure 1).

After opening a program file, the user selects the main or subprogram and a pretty- 
printed code is displayed. In semi-automatic mode, the user selects Transform $\rightarrow$ Parallelize to find the parallel loops. When automatic parallelization fails, program and loop restructuring filters are available. These include: loop unrolling and unimodular parallelization[13]. Also a link is offered to the loop restructuring Tiny tool developed by M. Wolfe[30]. However, in the presence of GOTO's and non-structured branches, loop analysis fails, because execution trajectories interfere and the scope of a loop body is ill defined. Therefore, FPT has a program restructurer which removes forward or backward branches and converts them into while loops. Next the while loops may be converted into do-loops by converting the induction variables into a function of the loop index. Finally, the resulting do-loops are tested for parallel iterations. These program transformations are described in detail in section 4.

In the graphic pane, the user views the call graph, the dependence graph, the task graph and the iteration space. The graph is kept synchronized with the program segment displayed. E.g. clicking a node or a dependence arc in the dependence graph will highlight the corresponding statements in the program.

\section{Dependence analysis}

\subsection{Analytical tests}

Dependences are analyzed using the Banerjee tests[8], taking into account if-statements, trapezoidal loop boundaries, and constant propagation where possible.

Example 1 shows the parallelism found in the Gauss-Jordan program, taking into account the if-test.

Besides the automatic detection of parallel loops, the user can ask specific dependence information, e.g. the dependences between statements and variables, the dependence type ( $\mathrm{a}=$ anti, $\mathrm{f}=$ flow, $\mathrm{o}=$ output) and the direction vector. Dependences occur when a variable is written $(W)$ and read $(R)$ in the same $(=)$ or different $(<,>)$ iterations. The sequence of $W$ and $R$ determines the dependence type: flow $(W \rightarrow R$ ), output $(W \rightarrow W)$ or anti $(R \rightarrow W)$. The direction vector indicates the iteration order for each dependence, e.g. a dependence between iterations $(2,3,4)$ and $(5,6,4)$ is indicated by a direction vector $(<,<,=)$. If the order cannot be determined, the corresponding loop index is $(*)$. 


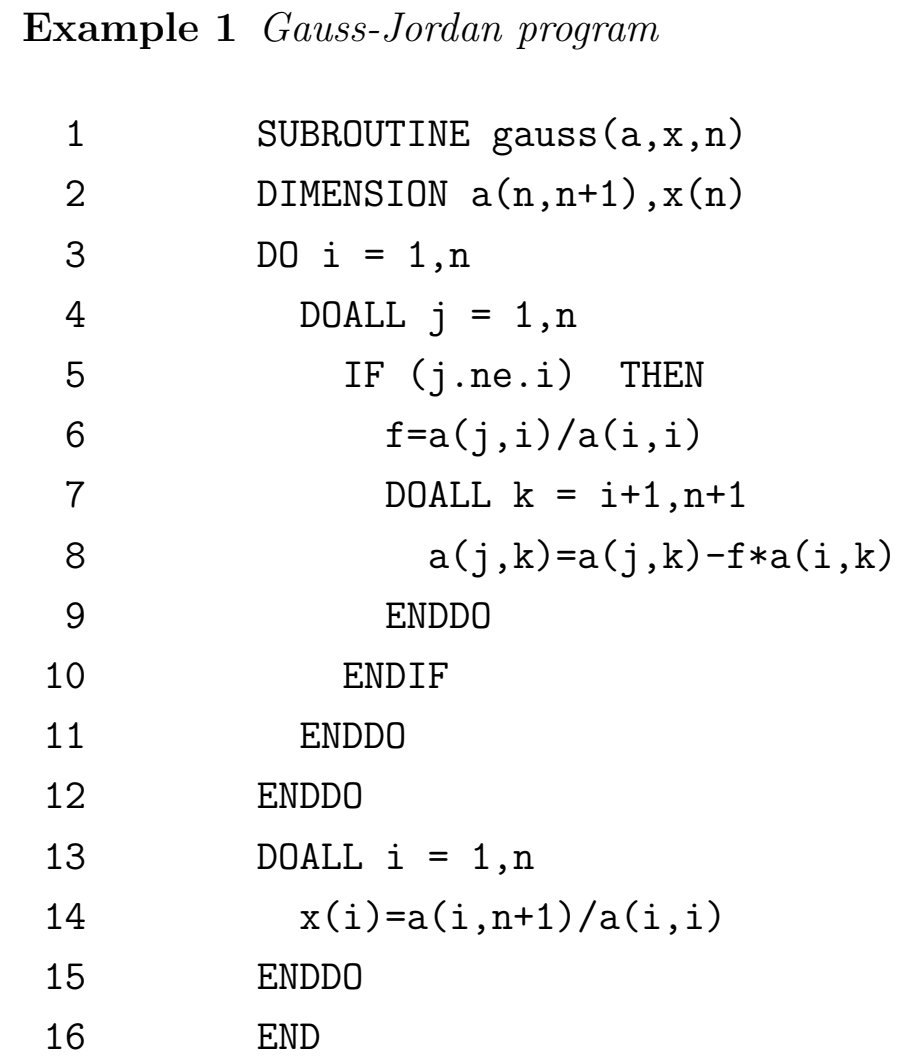

\subsection{Iteration space graph}

In many real programs the dependence relations are far from obvious. In this case, analytical methods fail or are too conservative for an efficient loop parallelization. Furthermore, the huge list of dependences and the dependence graph become intractable. In PEFPT the iteration space graph (ISG) is used as a complementary tool which gives detailed dependence information in an easy graphical form. This allows the user to recognize parallel loops and safely insert parallelizing directives. In a single loop, the ISG shows the dependences between statements. In a nested loop, the ISG represents the dependences between the iterations. Flow-, anti- and output-dependences are shown by red, blue and green edges respectively. Black nodes in the rectangular iteration region indicate that the corresponding iteration is executed. If the dependences can be determined analytically, the ISG is calculated, otherwise, the ISG is built from an instrumented program run. 


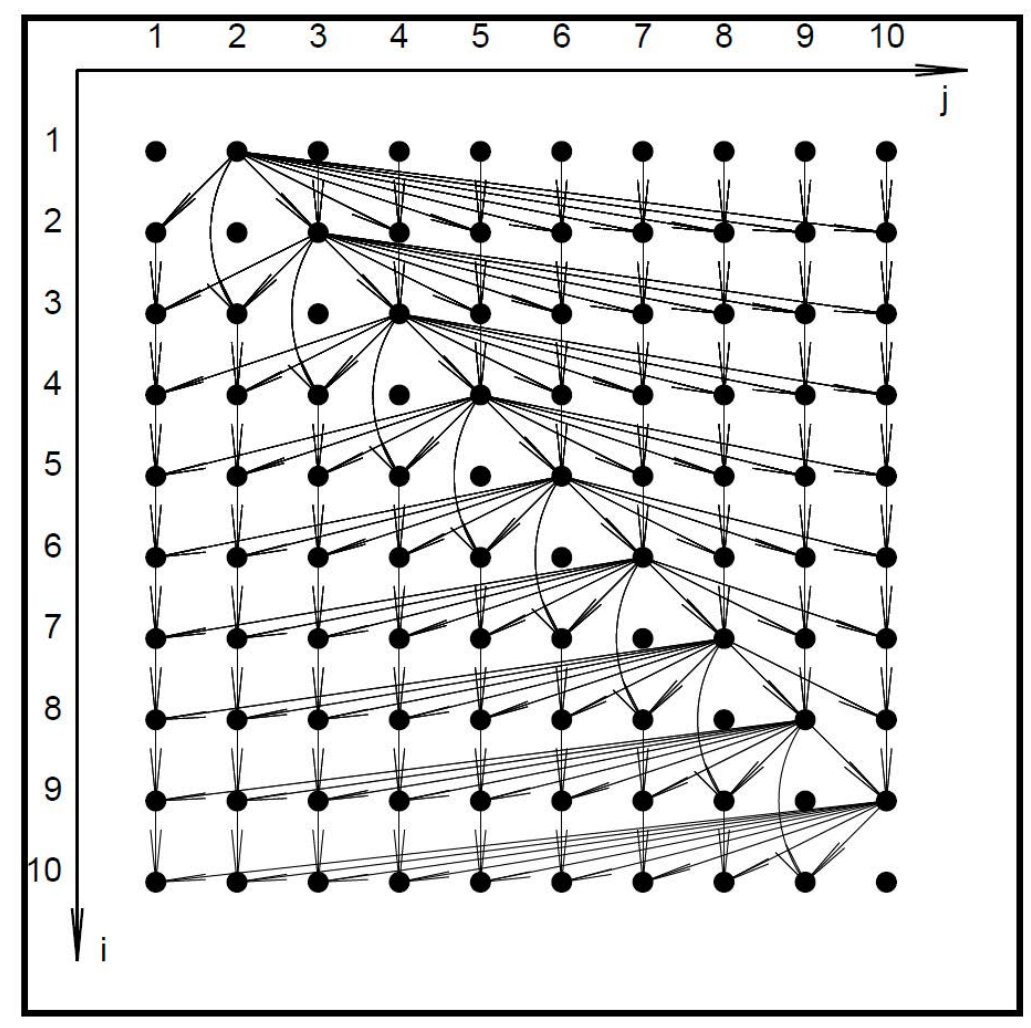

Figure 2: Iteration space graph of example 1 showing the flow dependences of array a in the area $(i, j) \in(1: 10,1: 10)$.

In a nested loop, the ISG shows the dependences between iterations. Consider the Gauss-Jordan elimination program in example 1. The flow dependences of variable a occurring in loops $i$ and $j$ are shown in figure 2. From this iteration space graph, it appears that all flow dependences of variable a are directed from lower to higher values of $i$. Since there are no flow dependences between the iterations $j$ for any particular $i$, all iterations in the $j$ loop are independent and can be executed in parallel. Note that the dependences of the $k$ loop are projected onto the $(i, j)$-plane. Consequently, the ISG takes also into account possible dependences in the $(i, j)$-plane generated from the inner loop $k$. Scalar $f$ generates dependences between the iterations of the $j$-loop. However, $f$ can be privatized per processor, and thus loop $j$ is a DOALL. Likewise the iteration space graph of the $(j, k)$-plane shows that there is no dependence between the $\mathrm{k}$-iterations, and therefore loop $\mathrm{k}$ is also a DOALL. This is also found by automatic parallelization, as shown in the example program 1. The Fast Fourier Transform program is a prominent example where the dependence analysis fails to recognize parallel loops and the iteration space graph helps the user to recognize the parallel loops. This example is discussed in section 6.1. 


\section{Program transformations}

FPT contains some well known loop transformations, such as loop unrolling, loop exchange and unimodular transformations. These transformation assume that a loop is block-structured and contains no entering or leaving branches. However, frequently real programs contain open-ended branches characterized by goto or if-goto statements. Usually the presence of these non-structured control statements prevents any further parallelization. Therefore a method has been devised and implemented to restructure the program into well-defined regions of control.

\subsection{Removal of ill-structured code}

Non-structured programs are difficult to parallelize. First, the scope of the nonstructured statements may interact and the execution trajectories can overlap, preventing the unique determination of the scope of a control statement. Second, even if the scope of a non-structured statement is determined and converted into a while-loop, this while-loop is still difficult to parallelize due to the loop carried dependences of the control variables. Therefore the program is restructured by stepwise transforming each hammock subgraph of the program control flow graph. In this way, non-structured statements are converted into block-if-statements and while-loops. Furthermore, an algorithm is developed to transform a common type of while-loops into do-loops. This technique works for while-loops of which the control variables appear as a linear recurrence relation. As a result, the loop carried dependences generated by the control variables are removed. If there are no other loop carried dependences, the do-loop may then be converted into a doall-loop. The correctness of each basic program transformation has been verified using an axiomatic inference system[34].

A lot of related work has been done on program restructuring. These techniques are applied either to reducible graphs [7, 23, 27, 28] or to irreducible graphs [6]. New variables are introduced to register the state of the condition of an if-goto statement. The newly proposed technique focuses on the discussion of forward, backward and exit branches. There is no distinction between processing a reducible or an irreducible flow graph. Furthermore, the hammock transformation minimizes the number of replications and new variables in irreducible flow graphs. 


\subsection{Hammock graph transformations}

There exist several approaches to restructure programs and many unstructured program forms have been discussed in the literature. However, principally there are two types of branches: backward and forward. Because the goto's which jump out of one or more loops are different from backward and forward branches, we call those goto's exit-jump.

Informally, a hammock graph [17] is a region of the program bounded by two statements, $n_{0}$ and $n_{e}$, such that all possible execution trajectories enter the hammock graph at its initial statement $n_{0}$ and leave it at the terminal statement $n_{e}$. Consequently, a correct transformation of the hammock graph maintains the correctness of the whole program. We define the following:

Definition 1 Branch and target.

A branch is a control statement of the following type:

$$
[\text { if }(<\exp >)] \text { goto }<\text { label }>
$$

The $<$ label $>$ is the target of the branch.

If a branch is lexically preceding its target, it is called a forward branch. Otherwise, it is called a backward branch.

Definition 2 Scope of an unstructured branch, $S C\left(i_{b}\right)$.

The scope of an if-statement, $S C\left(i_{b}\right)$, is the block of statements between the ifstatement and the target, excluding "if" and including the "target".

Definition 3 Interacting branches.

When two branches and its targets overlap, the branches interact, i.e. their scopes intersect:

$$
S C\left(i_{b}\right) \cap S C\left(j_{b}\right) \neq 0
$$

Definition 4 Hammock graph of a branch $i_{b}, H G_{i_{b}}$

A hammock graph of a branch is the union of all scopes which directly or indirectly overlap with $S C\left(i_{b}\right)$. A hammock graph has a single entry point $n_{0}$ and a single exit point $n_{e}$. 
Corollary: A program transformation which preserves the semantics of a hammock graph preserves the semantics of the whole program.

The branches are removed according to their type, in the order: backward branches, long exit jumps and forward branches.

A hammock transformation consists of finding a hammock graph in which the branches interact, then applying three code transformations, namely Backward-Copy, Cut and Forward-Copy. These transformations remove all non-structured branches inside the hammock graph. The process is repeated for all hammock graphs with interacting branches. Now the basic code transformations are described in detail.

\subsubsection{Backward-Copy and Cut Transformations}

Backward-Copy. Consider figure 3. Let $i_{b}$ be an arbitrary backward branch. A backward branch can be represented by a repeat-until statement, if there are no other branches jumping into the repeat loop. An incoming branch prevents the backward branch from being directly converted into a loop. However, since the body of a repeatuntil loop is executed at least once, the loop body can be unrolled once, and all the incoming branches are moved out of the loop. Then the backward branch is converted into a while-loop. As the figure 3 illustrates, after unrolling the loop, the targets of the incoming forward and backward branches $n_{0}$ and $n_{e}$ are moved outside the loop and the backward branch $i_{b}$ is converted into a while-loop containing no branch targets. This process of converting backward branches is known as Backward-Copy.

Order of branch elimination. One cannot take an arbitrary backward branch and use the Backward-Copy in order to eliminate this branch. For example in figure 3, branch $i_{b}$ is converted first. Branch $n_{e}$ can not be converted into a loop before $i_{b}$, because this would create a syntactically incorrect loop overlap. Branch elimination in a hammock graph therefore always starts from the initial branch, which is defined as follows.

Definition 5 Initial forward (backward) branch $i_{b}$ of a hammock graph $H G_{i_{b}}$.

The initial forward (backward) branch $i_{b}$ is the first forward (backward) branch in $H G_{i_{b}}$. 


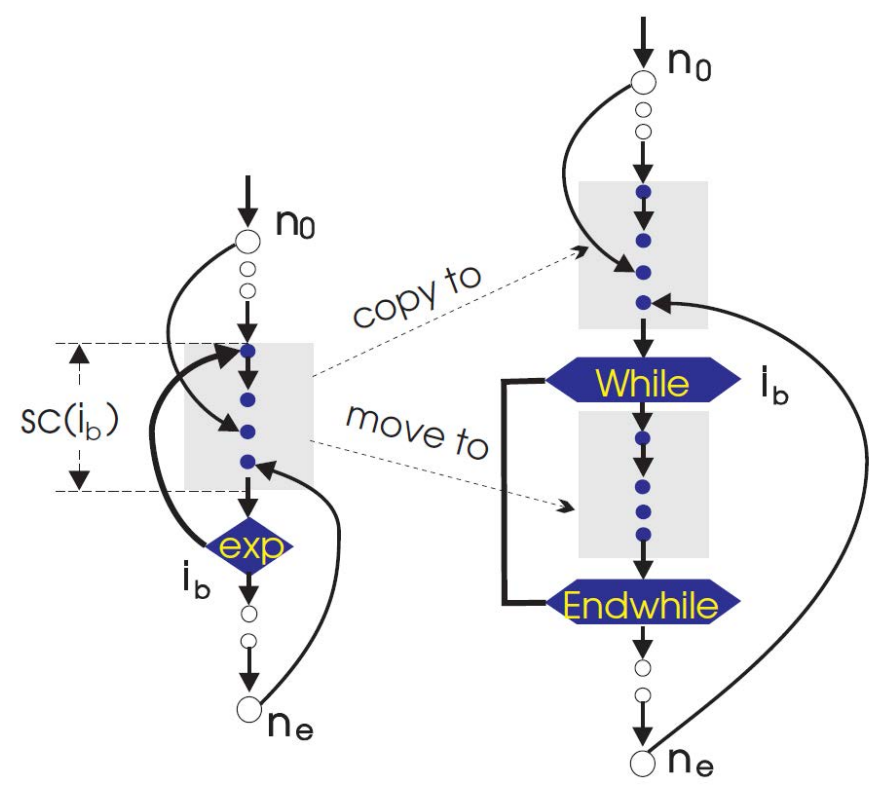

(a)

(b)

Figure 3: Removal of interacting backward branches. (a) The hammock graph $H G_{i_{b}}$ of backward branch $i_{b}$ with beginning and end nodes $n_{0}$ and $n_{e} . S C\left(i_{b}\right)$ is the scope of branch $i_{b}$. (b) After converting $i_{b}$ into a while-statement, the targets of $n_{0}$ and $n_{e}$ are moved outside the loop.

E.g. backward branch $i_{b}$ precedes $n_{e}$ and is therefore the initial backward branch of the surrounding hammock graph, $H G_{i_{b}}$. When branch $i_{b}$ is the initial backward branch, there are no other backward branches going out of its scope, $S C\left(i_{b}\right)$. Since there are no preceding backward branches, the Backward-Copy transformation creates no interaction with other backward branches. Furthermore, by construction, the targets of all other branches are moved outside the while-loop.

Next, the second initial backward branch is selected and eliminated. This procedure is repeated until there are no more backward branches.

Code duplication. In order to structure the program, some code duplications are needed when the control graph is irreducible. Consider the program (2.a). The scope of initial backward branch $\mathrm{S} 5, S C(\mathrm{~S} 5)$, is S3-S4. However, since S4 is the target of incoming forward branch $\mathrm{S} 1$, the scope $S C(\mathrm{~S} 5)$ is duplicated and a while-loop is generated around S3' and S4' (see (2.b)). Then the next backward branch S7 is converted into a repeat-until loop and finally the forward branch S1 reverts to a blockif (see next section). 
Example 2 Cut transformation

\begin{tabular}{|c|c|c|c|c|c|}
\hline $\mathrm{S} 1$ & IF (P1) & GOTO 200 & \multirow{2}{*}{\multicolumn{2}{|c|}{$\begin{array}{l}\text { S1 } \\
\text { S2 }\end{array}$}} & IF (.not.p1) \\
\hline $\mathrm{S} 2$ & \multicolumn{2}{|l|}{$\mathrm{S} 1=1$} & & & $\mathrm{~s} 1=1$ \\
\hline S3 100 & \multicolumn{2}{|l|}{$\mathrm{S} 2=2$} & S3 & 100 & $s 2=2$ \\
\hline S4 200 & \multicolumn{2}{|l|}{$\mathrm{S} 3=3$} & & & ENDIF \\
\hline S5 & IF (P2) & GOTO 100 & & 200 & REPEAT \\
\hline S6 & \multicolumn{2}{|l|}{$\mathrm{S} 4=4$} & \multicolumn{2}{|l|}{ S4 } & s3 $=3$ \\
\hline \multirow[t]{6}{*}{$\mathrm{S} 7$} & \multirow[t]{6}{*}{ IF (P3) } & \multirow[t]{6}{*}{ GOTO 200} & \multicolumn{2}{|l|}{ S5 } & WHILE (p2) \\
\hline & & & S3 & 15 & $s 2=2$ \\
\hline & & & S4 & 20 & $\mathrm{~s} 3=3$ \\
\hline & & & & & ENDDO \\
\hline & & & S6 & & $s 4=4$ \\
\hline & & & $\mathrm{S} 7$ & & UNTIL (p3) \\
\hline
\end{tabular}
(a) interacting forward and
(b) branches removed backward branches

Backward branch optimization. If the scope of a backward branch $S C\left(i_{b}\right)$ contains no incoming branches, the branch can be replaced by a repeat-until loop, without duplicating the scope $S C\left(i_{b}\right)$ in front of the loop.

Cut Transformation. After eliminating the backward branches, there may be loops with forward branches jumping out of the newly created while-loops. In order to remove these branches, the Cut conversion is applied as follows: 1) a new variable $b r_{i}$ is used to register the state of the branch condition and the loop control expression is modified by adding the new variable $b r_{i} ; 2$ ) the long jump is cut into two parts, one starts within the loop and jumps to the end of the loop, using EXIT, which is similar to a break statement in $\mathrm{C}$. The other is located outside the loop and jumps to its original target. See example 3 . 
Example 3 Long exit branch

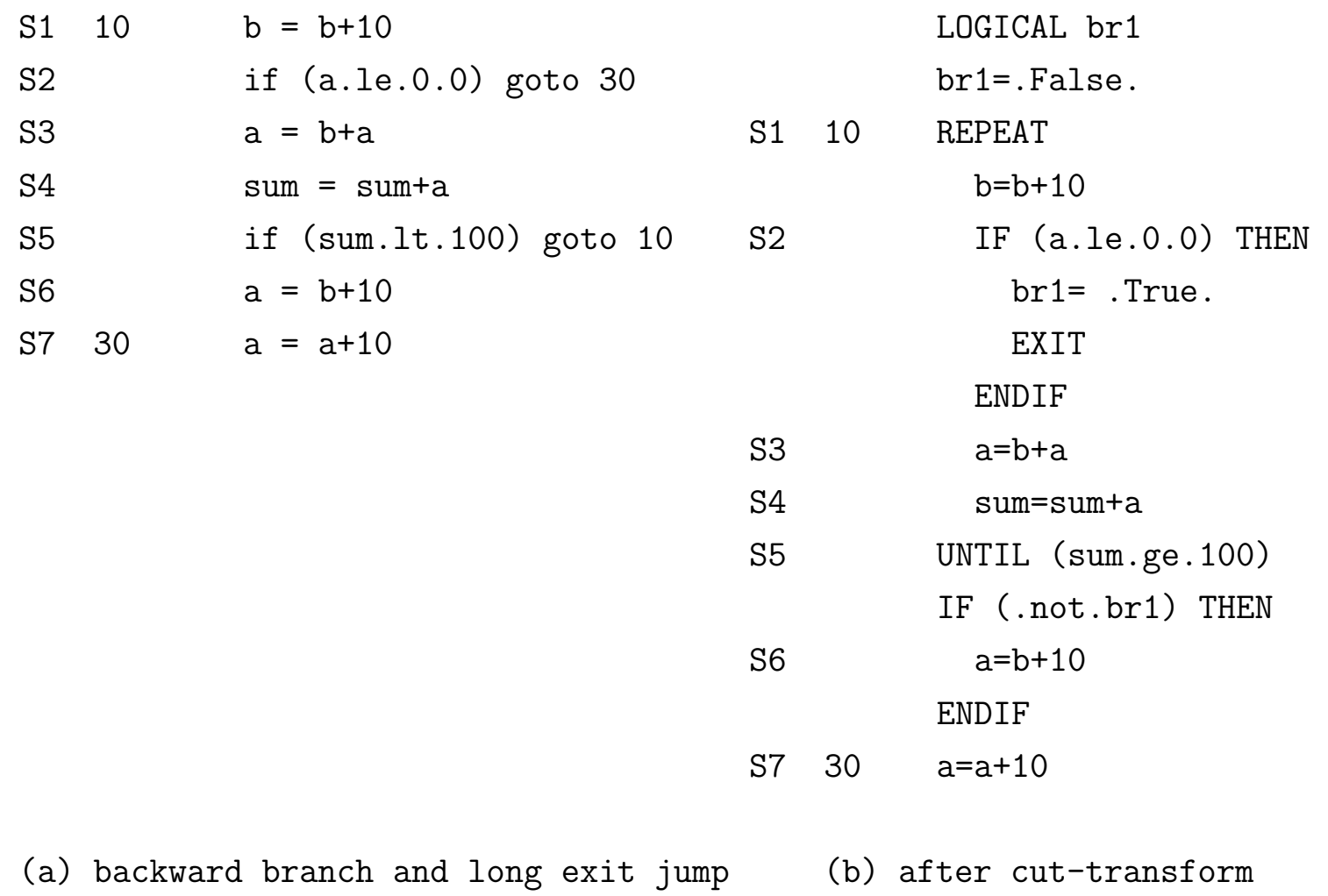

Statements S1-S5 constitute a backward loop in program in program (3.a). Because there is an outgoing forward branch $\mathrm{S} 2$, the termination of the loop depends on both if-statements S2 and S5. The Backward-Copy algorithm first converts the initial backward branch S5 into a repeat-statement. Since there are no incoming targets of other branches, the scope $S C(\mathrm{~S} 5)$ is not copied. To eliminate the exit-jump, a new temporary variable br 1 with the initial state .false. is created, which registers the state of the forward branch condition. The forward branch is then cut into two parts, as illustrated in example (3.b). Note that the EXIT can also be replaced by a GOTO to the first statement after S5.

\subsubsection{Forward-Copy Transformation}

In this section it is assumed that the backward branches have been removed from the program. 


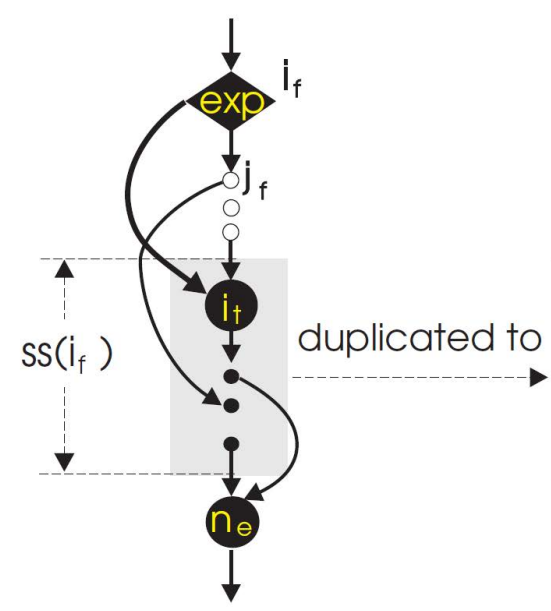

(a)

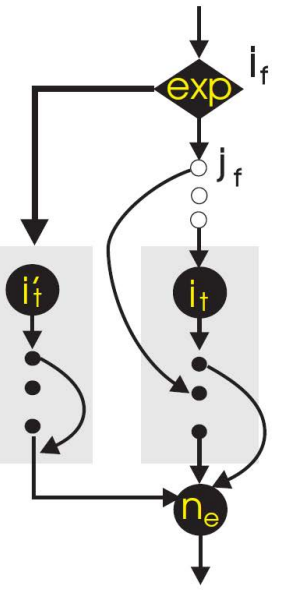

(b)

Figure 4: Removal of interacting forward branches. (a) $I_{f}$ is a forward branch with target $j_{t}$. The surrounding hammock graph $H G_{i_{f}}$ has entry node $i_{f}$ and terminal node $n_{e}$. The shaded region $S S\left(i_{f}\right)$ indicates the shared statements. (b) The shared statements are duplicated into the true part of $i_{f}$.

\section{Example 4 Forward-Copy}

\begin{tabular}{|c|c|c|c|c|}
\hline $\mathrm{S} 1$ & IF (J.LT.N) GOTO 60 & S1 & & IF (j.lt.n) \\
\hline S2 & $A=B+I$ & S4 & \multirow[t]{2}{*}{15} & $\mathrm{a}=\mathrm{b}+\mathrm{a}$ \\
\hline S3 & IF (A.GT.10) GOTO 80 & S5 & & $b=b-1$ \\
\hline 60 & $A=B+A$ & & & ELSE \\
\hline S5 & $B=B-1$ & $\mathrm{~S} 2$ & & $a=b+i$ \\
\hline \multirow[t]{6}{*}{ S6 } & $B=A-5$ & S3 & & IF (a.le.10) \\
\hline & & $\mathrm{S} 4$ & \multirow[t]{4}{*}{60} & $a=b+a$ \\
\hline & & S5 & & $b=b-1$ \\
\hline & & & & ENDIF \\
\hline & & & & ENDIF \\
\hline & & s6 & & $b=a-5$ \\
\hline
\end{tabular}

$\begin{array}{ll}\text { (a) forward branches } & \text { (b) after branch removal }\end{array}$ 
Duplication of shared statements. A forward branch transfers the control flow according to a Boolean condition. The first path is called the true_part while the other is called the false_part. Assume that an initial forward branch $i_{f}$ interacts with an other forward branch (see figure 4). The statements on the path between the target of $i_{f}$ and the terminal node $n_{e}$ are called the shared statements, $S S\left(i_{f}\right)$. Forward branch $i_{f}$ is converted into a block-if by duplicating the shared statements into the true_part. This process is called Forward-Copy.

To illustrate the method, refer to the program (4.a). The two forward branches S1 and S3 interact. The shared statements S4, S5 are duplicated to S4', S5' as the true part of S1, while the statements S2, S3, S4, S5 are located in the false part of S1. After the Forward Copy, both block-if's are hammock graphs (see the program (4.b) and figure 4).

\subsection{Converting a while-loop into a do loop}

$\mathrm{Y} . \mathrm{Wu}[32]$ has formalized a while-loop as follows:

$$
\begin{aligned}
& \text { while } b(T) \\
& \begin{array}{c}
T=g(T) \\
U=h(D, T)
\end{array} \\
& \text { endwhile }
\end{aligned}
$$

Here $T$ is the set of variables controlling Boolean condition $b(T)$ and $D$ are the other variables. $U$ is the set of output variables, excluding $T$. The control variables $T$ are modified by the function $g$ and the data are modified by the function $h$. A whilestatement establishes a loop of which the Boolean expression $b(T)$ is modified during an iteration. Therefore, the number of iterations depends on $b(T)$. The key to the translation of while-loops into do-loops is to determine the number of iterations. This requires that $b(T)$ can be represented as a function of a loop counter $k$. If $b(T)=b_{f}(k)$, the number of iterations $N_{i t}$ is the smallest integer which makes $b_{f}\left(N_{i t}\right)=$ false. As a result, the while-loop (1) is converted into a do-loop by replacing while $b(T)$ by do $k=1, N_{i t}$.

When the induction statements $T=g(T)$ create loop carried dependences, the resulting do-loop cannot be parallelized. Often the induction statements represent a set of coupled linear recurrence relations, for which an analytical solution exists. In this 
case FPT converts the control variable $T$ into a function $f(k)$ of the loop counter $k$. As a result, the loop carried induction dependence is eliminated. Next, the Boolean expression $b(T)$ is represented as a function $b_{f}(k)$ by substituting $f(k)$ into $b(T)$, where $b_{f}=b \circ f$. Finally, the number of iterations $N_{i t}$ is found by calculating the first integer value of $n$ such that $b_{f}(n)=$ false. The number $n$ can be determined by a binary search, or solved explicitly when the Boolean function is of the form $f(n) \geq 0$, such as in the FFT-program (see section 6.1). If there are no other loop carried dependences, the loop is converted into a DOALL-loop.

\subsection{Loop transformations}

When the array subscripts are linear and differ only by a constant, a unimodular transformation exists which generates outermost DOALLs with a large granularity[13]. The available loop transformations in the interactive mode are extended by a link to the well-known Tiny-tool developed by Michael Wolfe [30]. The user can select and clip any particular loop or nested loop, edit, modify and apply the loop transformations such as interchange, skewing, strip mining, wavefront as well as unimodular transformations. In each step the user can perform a dependence test and verify the parallelism gained by the transformation.

\section{$5 \quad$ PVM interface}

\subsection{Code generation}

After detecting the parallel loops, FPT generates code for different platforms, i.e. Fortran/MP for Sun Sparc, assembly code for a multiprocessor prototype (VPS[14]), threaded-code and PVM. The PVM software $[15,18]$ creates a Parallel Virtual Machine on a network of workstations. Basically PVM consists of a C- or Fortran-callable message passing library and a communication daemon which greatly facilitates the execution of a parallel application on distributed computers. However, users still need to find the parallelism and to program the data exchange and the task synchronization explicitly. During the process, new errors may be introduced when a sequential program is translated into the PVM code. Recently, a few tools have been developed for generating PVM code from a user's application. 
ADAPTOR of GMD [11] is an Automatic DAta Parallelism TranslatOR for transforming data parallel programs written in Fortran with array extensions, parallel loops, and layout directives to parallel programs with explicit message passing. It supports the data parallel languages Connection Machine Fortran and High Performance Fortran (HPF). The user has to express the parallelism explicitly using HPF statements. The resulting code is linked with the Adaptor libraries, which hide the PVM-code.

PARADDAPT of Washington state university [24] is another compilation system which converts a sequential program to parallel PVM code. PARADDAPT consists of several cooperating tools i.e. the Parafrase-2 parallelizing compiler, a data distribution tool ADDT, and a HPF compiler, ADAPTOR. Sequential programs are parallelized into parallel PVM codes using the HPF code generator. Here the user may use Parafrase to analyze and parallelize the loops.

FPT, the Fortran Parallel Transformer developed at the University of Ghent, is an integrated interactive parallel programming environment for parallelizing programs. Besides the parallelization tools, the generation of PVM code is simple and transparent to the user. Furthermore, no extra libraries or tools are needed, apart from the public domain PVM system. The PVM-code generator of FPT converts the serial program into master and slave programs. The parallel loops are partitioned to be executed by the number of available servers. The communication is optimized by using a data broadcast from the master to all slaves and by minimizing the amount of data sent.

A PVM application consists of a master program and several slave programs. Each outer DOALL loop found by FPT constitutes a separate job executed by the slaves. Therefore the $N$ independent iterations are partitioned over the $p$ processors in bands of $\lceil N / p\rceil$ iterations. The granularity can be refined by selecting $p>p_{\max }$, where $p_{\max }$ is the maximum number of available processors for the job.

Each parallel loop is executed in three steps.

1. In the prologue, the input data for all slaves is gathered and put into a single message. The number of iterations in one task is calculated and included in the message. Finally, the sequence number of every slave is added. Then this message is broadcast. 
2. In the execution phase, the slave program unpacks the message and executes the loop body corresponding to its sequence number sent by the master. During this phase there is no communication between the slaves, because the inner loop iterations are independent.

3. In the epilogue, each slave packs and sends back the results and the master stores the received data in the proper locations. Care is taken that the data results of each slave do not overlap.

\subsection{Data distribution}

Data packing. Let $l h s$ and $r h s$ be the data references at the left hand side and the right hand side of the assign statements in a parallel loop. Then the input data and the output data for a slave are $r h s$ and $l h s$ respectively. The input data $r h s$ is packed one by one in a message buffer using the command

$$
\text { call pvmfpack(type, name, } n \text {, stride, info). }
$$

Here type is a constant representing the variable type, name represents a scalar or an array element. As a result $\mathrm{n}$ elements at distance stride are added to the message buffer, info. Similarly, at the slave, the pvmfunpack routine unloads the message buffer. This PVM-call allows arrays with linear index expressions to be packed in a single call. E.g., the input for the following parallel loop

$$
\begin{aligned}
& \text { doall } i=1, n \\
& \quad b(i)=a(i)+c(2 * i) \\
& \text { enddo }
\end{aligned}
$$

is packed and broadcast to the slaves by

$$
\begin{aligned}
& \text { call pvmfpack( } 3, \mathrm{a}, \mathrm{n}, 1, \text { info }) \\
& \text { call pvmfpack( } 3, \mathrm{c}, \mathrm{n} / 2,2, \text { info) } \\
& \text { call pvmfmcast (info) }
\end{aligned}
$$


Broadcast of the input data. In the prologue of each job, the input data for all slaves is gathered into a single message. The message is sent only once using the pvmfmcast broadcast call. This saves significant communication overhead in a CSMA/CD Ethernet LAN, since the data is available at all processors simultaneously [25]. Unfortunately, the multicast call pvmfmcast, available in PVM since version 3.2.6 is still implemented as a sequential unicast to all slaves. A true multicast PVM daemon contains the pvm_ipmcast multicast call, which allows a group of LAN-machines to receive each others broadcast messages. Recent studies show that reliable IP multicast works well in specially tailored LAN protocols $[12,16]$. However, a general implementation of multicast in PVM is not foreseen in the near future because reliable IP multicast is not generally available. This is due in part to the lack of vendor support and the absence of a suitable RFC (request for comments).

Minimizing overlap. Redundant data communication may be generated when data areas overlap. First data overlap is defined and then the packing of overlapping data is minimized.

It is assumed that the array subscripts are linear index expressions and that the loops are normalized with rectangular bounds and a step size one. Under these assumptions a reference of array $A$ with $n$ dimensions has the form $A\left(e_{1}, \ldots, e_{n}\right)$, where $e_{k}=d_{k} i_{k}+c_{k}$ is the index expression in the $k$-th dimension, $1 \leq k \leq n$.

\section{Definition 6 Data area.}

Let $s_{k}=\left|d_{k}\right|$ be the stride by which a reference of array $A$ is accessed. Denote by $l_{k}$ and $u_{k}$ the lower and upper bounds of index expression $e_{k}$, i.e. $l_{k} \leq u_{k}, \forall k$. Then the data area covered by the reference of $A$ in the loop is a rectangular region denoted as follows:

$$
\operatorname{Area}\left(A\left(e_{1}, \ldots, e_{n}\right)\right) \stackrel{\text { def }}{=}\left(l_{1}: u_{1}: s_{1}, \ldots, l_{n}: u_{n}: s_{n}\right)=(\mathbf{L}: \mathbf{U}: \mathbf{S})
$$

where $\mathbf{L}=\left(l_{1}, \ldots, l_{n}\right), \mathbf{U}=\left(u_{1}, \ldots, u_{n}\right)$ and $\mathbf{S}=\left(s_{1}, \ldots, s_{n}\right)$.

Equation (2) can also be written as

$$
\operatorname{Area}\left(A\left(e_{1}, \ldots, e_{n}\right)\right)=\left\{A\left(\mathbf{D}_{\mathbf{s}} \mathbf{I}+\mathbf{L}\right) \mid \mathbf{I}=\left\{i_{k}\right\} \text { with } 0 \leq i_{k} \leq\left\lfloor\left(u_{k}-l_{k}+1\right) / s_{k}\right\rfloor\right\}
$$


Here $\mathbf{D}_{\mathbf{s}}$ is a constant diagonal matrix with elements the stride vector $\mathbf{S}=\left(s_{1}, \ldots, s_{n}\right)$.

Definition 7 Overlapping data areas.

Two areas $A_{1}$ and $A_{2}$ overlap if they have at least one common element.

FPT checks the following sufficient overlap conditions:

Lemma 1 Sufficient overlap conditions.

Let $A_{1}=(\mathbf{L}: \mathbf{U}: \mathbf{S})$ and $A_{2}=\left(\mathbf{L}^{\prime}: \mathbf{U}^{\prime}: \mathbf{S}^{\prime}\right)$ be two data areas. $A_{1}$ and $A_{2}$ overlap if the following conditions are true:

$$
\begin{aligned}
& \max \left(\mathbf{L}, \mathbf{L}^{\prime}\right) \leq \min \left(\mathbf{U}, \mathbf{U}^{\prime}\right) \\
& \operatorname{gcd}\left(\mathbf{S}, \mathbf{S}^{\prime}\right) \text { divides }\left|\mathbf{L}-\mathbf{L}^{\prime}\right|
\end{aligned}
$$

and

$$
\operatorname{lcm}\left(\mathbf{S}, \mathbf{S}^{\prime}\right) \leq \min \left(\mathbf{U}, \mathbf{U}^{\prime}\right)-\max \left(\mathbf{L}, \mathbf{L}^{\prime}\right)
$$

Here the operators max, min, gcd (greatest common divisor) and lcm (least common multiple) operate on pairwise elements of the two vector arguments, e.g.

$$
\max \left(l_{k}, l_{k}^{\prime}\right) \leq \min \left(u_{k}, u_{k}^{\prime}\right), \forall k
$$

Proof: Assume condition (4) is not true. Then there exists a bound $k$ such that $\max \left(l_{k}, l_{k}^{\prime}\right)>\min \left(u_{k}, u_{k}^{\prime}\right)$. Without loss of generality, suppose $l_{k}=\max \left(l_{k}, l_{k}^{\prime}\right)$. Since by definition $6, l_{k} \leq u_{k}$, one has $l_{k}>u_{k}^{\prime}$ or $e_{k}>e_{k}^{\prime}, \forall k$, and $A_{1}$ and $A_{2}$ cannot have common elements in dimension $k$. Therefore condition (4) is necessary. Furthermore, in order to $A_{1}$ and $A_{2}$ have at least one element in common, a solution of the set of $n$ Diophantine equations $e_{k}=e_{k}^{\prime}, \forall k$, must exist. In other words, there exist integer indices $\mathbf{I}$ and $\mathbf{J}$ such that

$$
\mathbf{D}_{\mathbf{s}} \mathbf{I}+\mathbf{L}=\mathbf{D}_{\mathbf{s}^{\prime}} \mathbf{J}+\mathbf{L}^{\prime}
$$

Since $\mathbf{D}_{\mathbf{s}}$ and $\mathbf{D}_{\mathbf{s}^{\prime}}$ are diagonal matrices, the Diophantine equations (7) are independent. An integer solution exists iff $\operatorname{gcd}\left(\mathbf{S}, \mathbf{S}^{\prime}\right)$ divides $\left|\mathbf{L}-\mathbf{L}^{\prime}\right|[8]$. This is condition (5). 
Finally, the integer solutions of equations (7) should belong to the rectangular areas $A_{1}$ and $A_{2}$ respectively. Consider the $k$-th Diophantine equation of (7):

$$
s_{k} i_{k}+l_{k}=s_{k}^{\prime} j_{k}+l_{k}^{\prime}
$$

With $\left(i_{0}, j_{0}\right)$ a particular integer solution of equation (8), the general solution is ([8])

$$
\begin{aligned}
& \left(\begin{array}{ll}
i_{k} & j_{k}
\end{array}\right)=\left(\left(l_{k}^{\prime}-l_{k}\right) / g \quad t\right)\left(\begin{array}{cc}
i_{0} & j_{0} \\
-s_{k}^{\prime} / g & -s_{k} / g
\end{array}\right) \\
& =\left(i_{0}\left(l_{k}^{\prime}-l_{k}\right) / g-t s_{k}^{\prime} / g \quad j_{0}\left(l_{k}^{\prime}-l_{k}\right) / g-t s_{k} / g\right)
\end{aligned}
$$

where $g=\operatorname{gcd}\left(s_{k}, s_{k}^{\prime}\right)$ and $t$ is an arbitrary integer. By substituting the solution $i_{k}$ and $j_{k}$ into equation (8) one finds that the overlapping data points are spaced at a constant interval $v_{k}$,

$$
v_{k}=s_{k} s_{k}^{\prime} / g=\operatorname{lcm}\left(s_{k}, s_{k}^{\prime}\right)
$$

In order to have at least one overlapping element in dimension $k$, it is sufficient that $v_{k}<\min \left(u_{k}\right)-\max \left(l_{k}\right)$ or

$$
\operatorname{lcm}\left(s_{k}, s_{k}^{\prime}\right)<\min \left(u_{k}\right)-\max \left(l_{k}\right)
$$

A solution to the set of Diophantine equations (7) exists if the inequality (11) holds for all $k$. This is expressed by condition (6).

Now define the shared region.

Definition 8 Shared region, $R$.

The shared region of two overlapping data areas $A_{1}=(\mathbf{L}: \mathbf{U}: \mathbf{S})$ and $A_{2}=\left(\mathbf{L}^{\prime}: \mathbf{U}^{\prime}\right.$ : $\mathbf{S}^{\prime}$ ) is defined as the $n$-dimensional cube

$$
R=\left(\max \left(\mathbf{L}, \mathbf{L}^{\prime}\right): \min \left(\mathbf{U}, \mathbf{U}^{\prime}\right): 1\right)=\left(\mathbf{L}^{\mathbf{r}}: \mathbf{U}^{\mathbf{r}}: 1\right)
$$

The maximal amount of data reduction equals the fraction $F$ of data points which overlap in the shared region, $R$. Therefore, denote by $T$ the total number of data points in the shared area $\left(A_{1} \cup A_{2}\right) \cap R$. $T$ is the product of the number of data points in each index, $k=1, \ldots, n$ and is approximated as follows: 


$$
\begin{aligned}
T & =\prod_{k=1}^{n}\left(\frac{\left(u_{k}^{r}-l_{k}^{r}+1\right)}{s_{k}}+\frac{\left(u_{k}^{r}-l_{k}^{r}+1\right)}{s_{k}^{\prime}}\right) \\
& =\prod_{k=1}^{n} \frac{\left(s_{k}+s_{k}^{\prime}\right)}{s_{k} s_{k}^{\prime}}\left(u_{k}^{r}-l_{k}^{r}+1\right)
\end{aligned}
$$

In region $R$, the coinciding data points of $A_{1}$ and $A_{2}$ are spaced at $\mathbf{S}^{\mathbf{r}}=\operatorname{lcm}\left(\mathbf{S}, \mathbf{S}^{\prime}\right)$. Let $V$ be the number of common data points in the shared region, $A_{1} \cap A_{2} \cap R$. One has

$$
V=\prod_{k=1}^{n} \frac{u_{k}^{r}-l_{k}^{r}+1}{s_{k}^{r}}
$$

The fraction of overlapping data points of $A_{1} \cap A_{2}$ in region $R$ is

$$
\begin{aligned}
F=V / T & =\prod_{k=1}^{n} \frac{s_{k} s_{k}^{\prime}}{s_{k}^{r}\left(s_{k}+s_{k}^{\prime}\right)} \\
& =\prod_{k=1}^{n} \frac{\operatorname{gcd}\left(s_{k}, s_{k}^{\prime}\right)}{s_{k}+s_{k}^{\prime}}
\end{aligned}
$$

with $\operatorname{gcd}\left(s_{k}, s_{k}^{\prime}\right) \times \operatorname{lcm}\left(s_{k}, s_{k}^{\prime}\right)=s_{k} s_{k}^{\prime}$.

For instance, if two one-dimensional arrays are spaced at strides 3 and 4 then they have an overlap fraction $F=1 / 7$. When two areas $A_{1}$ and $A_{2}$ have the same stride $s_{k}$ in all dimensions, the overlap fraction is $F=1 / 2$. This allows a data reduction of $F=50 \%$ in the shared region, by sending $A_{1} \cap R$ instead of $\left(A_{1} \cup A_{2}\right) \cap R$.

A data set containing all points of $A_{1}$ and $A_{2}$ in $R$ is area $H=\left(\mathbf{L}^{r}: \mathbf{U}^{r}: \operatorname{gcd}\left(\mathbf{S}, \mathbf{S}^{\prime}\right)\right)$. However, if $\operatorname{gcd}\left(\mathbf{S}, \mathbf{S}^{\prime}\right)<\min \left(\mathbf{S}, \mathbf{S}^{\prime}\right)$, then $H$ includes data not belonging to $A_{1}$ or $A_{2}$. In that case sending area $H$ increases the overhead and even may overwrite valid data in the slave computer. For this reason, data is merged only where the areas $A_{1}$ and $A_{2}$ fully overlap.

Definition 9 Fully overlapping data areas.

Two data areas $A_{1}$ and $A_{2}$ fully overlap if they overlap and furthermore one has

$$
\operatorname{gcd}\left(\mathbf{S}, \mathbf{S}^{\prime}\right)=\min \left(\mathbf{S}, \mathbf{S}^{\prime}\right)
$$




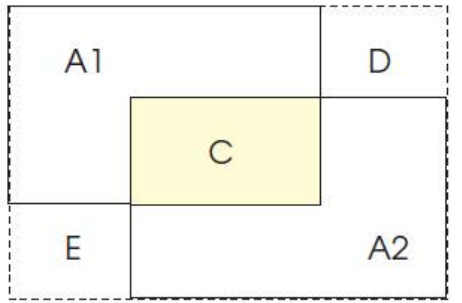

(a)

\begin{tabular}{|c|}
\hline$A 1$ \\
\hline$C$ \\
\hline$A 2$ \\
\hline
\end{tabular}

(b)

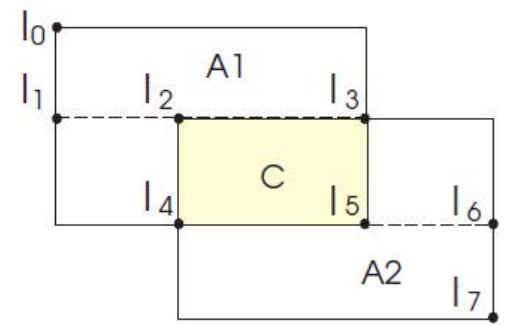

(c)

Figure 5: Areas overlap

In the shared region of fully overlapping areas, the area with the smallest stride is sent, since it includes the area with the largest stride. The fraction of redundant data in this case is:

$$
F_{\text {full }}=\prod_{k=1}^{n} \frac{\min \left(s_{k}, s_{k}^{\prime}\right)}{s_{k}+s_{k}^{\prime}} .
$$

Figure 5 gives some examples where areas A1 and A2 overlap in C. When A1 and A2 are merged, the data in $\mathrm{C}$ is sent only once. The non-overlapping areas are sent separately. The technique is illustrated by an example. A segment code of a slave is given by the following program.

\section{Example 5}

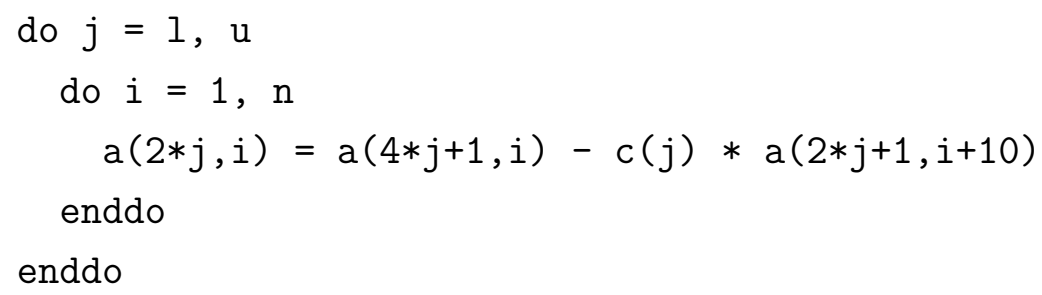

The input and output data for the slaves are determined by the $r h s$ and $l h s$ respectively. The left hand side covers area $(2 l: 2 u: 2,1: n: 1)$ of matrix $a$. The right hand side contains area $(l: u: 1)$ of array $c$ and two overlapping areas $A_{1}$ and $A_{2}$ of matrix $a$ (see figure 5.c):

$$
\begin{array}{ll}
A_{1}=\mathbf{I}_{0} \mathbf{I}_{5}=\operatorname{Area}(a(2 j+1, i+10)) & =(2 l+1: 2 u+1: 2,11: n+10: 1) \\
A_{2}=\mathbf{I}_{2} \mathbf{I}_{7}=\operatorname{Area}(a(4 j+1, i)) & =(4 l+1: 4 u+1: 4,1: n: 1)
\end{array}
$$


Areas $A_{1}$ and $A_{2}$ overlap when $n>10$. The overlap is full, since the strides $(2,1)$ $\left(A_{1}\right)$ and $(4,1)\left(A_{2}\right)$ satisfy equation $(12)$. By a merging operation, the input data area consists of three data regions:

$$
\begin{aligned}
& \mathbf{I}_{0} \mathbf{I}_{5}=(2 l+1: 2 u+1: 2,11: n+10: 1) \\
& \mathbf{I}_{3} \mathbf{I}_{6}=(2 u+1: 4 u+1: 4,11: n: 1) \\
& \mathbf{I}_{4} \mathbf{I}_{7}=(4 l+1: 4 u+1: 4,1: 11: 1)
\end{aligned}
$$

Since vector $\mathrm{c}$ is only input data, the master sends vector $\mathrm{c}$ only once. Using the input and output areas of matrix $a$, the generation of PVM code for sending and receiving data is straight forward.

\section{Results}

\subsection{Parallelizing the FFT kernel using the ISG}

The iteration space graph can help the programmer to find less obvious parallelism. Consider the FFT kernel in example 6. After restructuring the FFT kernel by removing the GOTO's and converting the resulting WHILE into a DO-loop, the program 6(b) results. Automatic parallelization is not able to detect parallel loops in this case. The flow dependences for the array data are shown in figure 6 for $\mathrm{n}=16$. It immediately becomes clear that all loop-carried dependences point from iteration $\mathrm{k} 1$ to iteration k1+1. Consequently, the k1 loop has to be executed sequentially. At the other hand, there are no dependences between the iterations of loop $i$ or loop $\mathrm{m}$ for a particular value of $\mathrm{k} 1$. Therefore, the $\mathrm{i}$ - and the $\mathrm{m}$-loops can be executed in parallel.

\subsection{PVM benchmark}

Generating PVM code is an important aspect of the FPT environment. PVM code is generated from a serial users' application first by extracting parallel loops via the data and control dependence analysis, next by partitioning the code and distributing the data to the slaves. Furthermore, redundant data communication is minimized by merging overlapping data areas.

Three examples, GJ (Gauss-Jordan Elimination), MM (Matrix Multiplication) and CONVOL, a convolution program, illustrate the use of FPT-generated PVM code. 
Table 1 gives speedups when problems with dimensions 400 and 800 are executed on 1 to 4 processors. The codes were executed on HP7XX workstations connected by an Ethernet $10 \mathrm{Mb} / \mathrm{s}$ network.

Example 6 Fast Fourier Kernel program
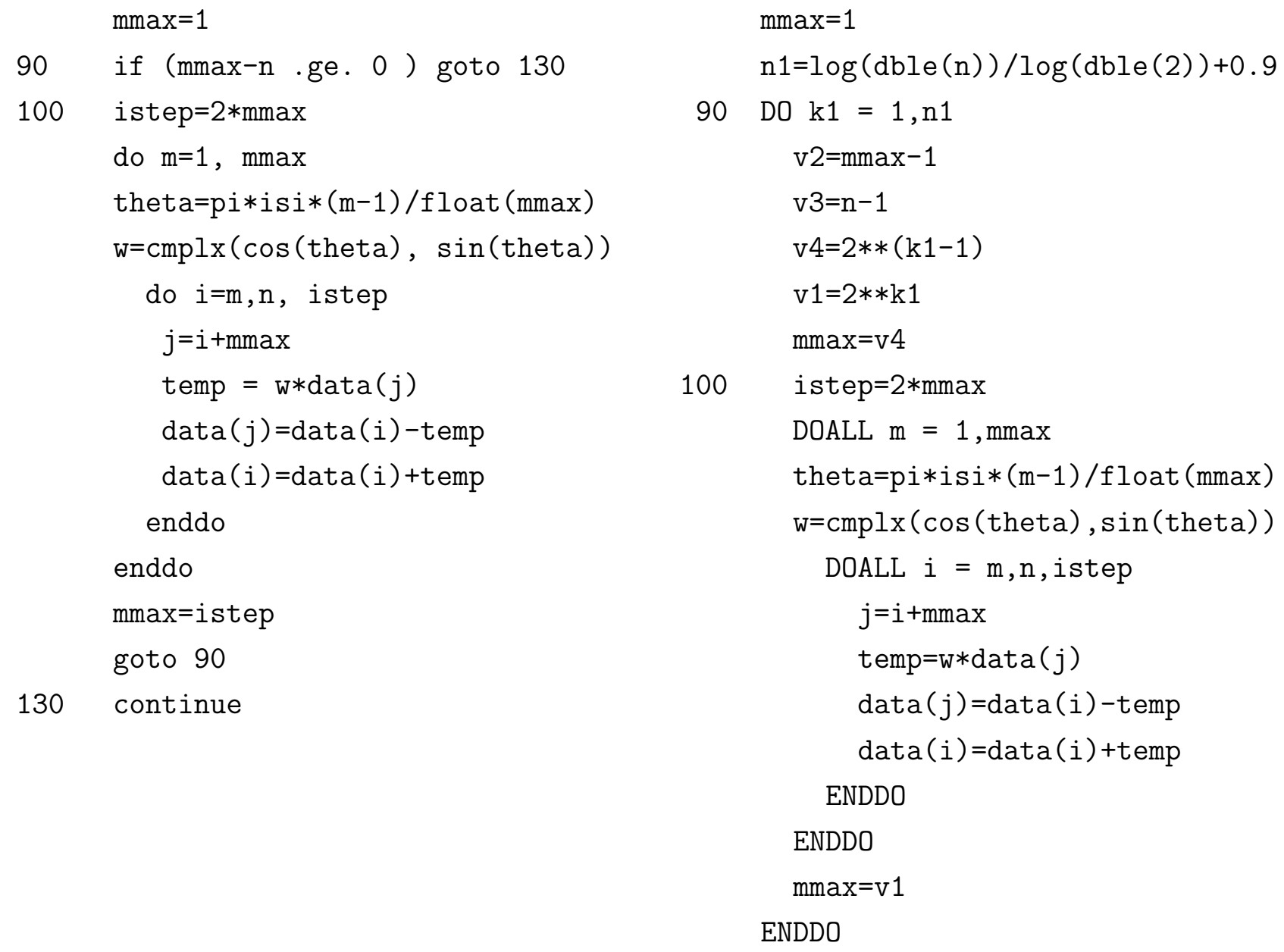

(a) original FFT kernel

(b) restructured FFT kernel 

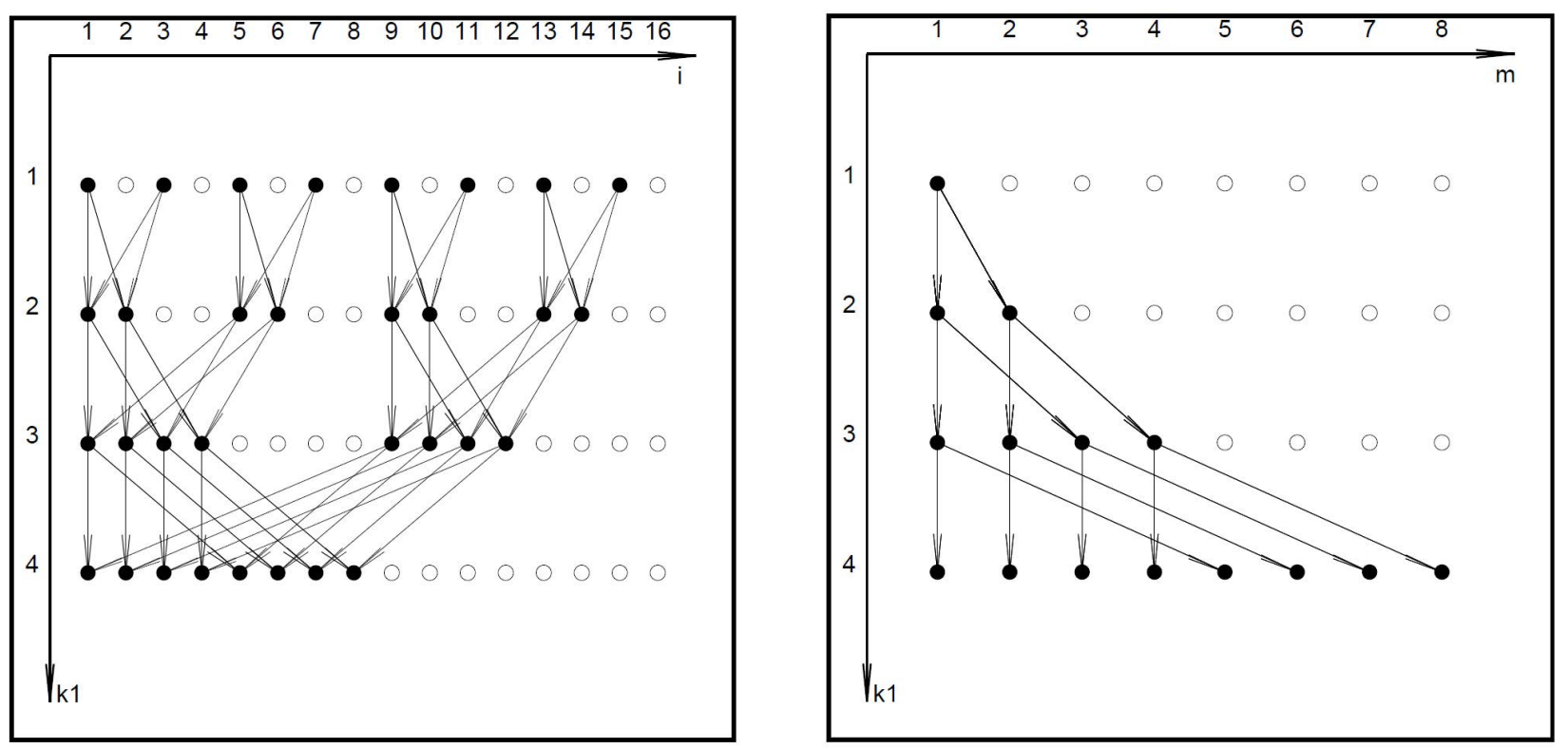

Figure 6: Iteration space graphs of nested loops $(\mathrm{k} 1, \mathrm{i})$ and $(\mathrm{k} 1, \mathrm{~m})$ in the restructured FFT kernel program (16 data points). Black nodes indicate the iterations executed. The arrows indicate the flow dependences of array data. The step size of the i-loop doubles in each $\mathrm{k} 1$-iteration, $\mathrm{n} 1=\log _{2}(n)$. There are no flow dependences between the i-nodes (left graph) and m-nodes (right graph) for each k1-iteration, therefore the i-loop and the m-loop are DOALL's. This assumes that the scalars in the loops are privatized for each processor.

\begin{tabular}{|c|c|c||c|c||c|c|}
\hline & \multicolumn{2}{|c||}{ GJ } & \multicolumn{2}{c|}{ MM } & \multicolumn{2}{c|}{ CONV } \\
\hline \#procs & 400 & 800 & 400 & 800 & 400 & 800 \\
\hline 1 & 1 & 1 & 1 & 1 & 1 & 1 \\
2 & 1.3 & 1.33 & 1.77 & 1.70 & 1.38 & 1.83 \\
3 & 1.7 & 2.48 & 1.77 & 1.70 & 1.12 & 2.92 \\
4 & 1.9 & 3.17 & 2.77 & 3.04 & 1.33 & 3.05 \\
\hline
\end{tabular}

Table 1: Speedups of 3 programs: Gauss-Jordan, Matrix Multiply and $3 \times 3$ kernel Convolution 


\subsection{PVM code generation for the Jacobi kernel}

The following example illustrates the PVM-code productivity gain. Consider the following 19-line Jacobi program as parallelized by FPT:

\section{Example 7 Jacobi kernel}

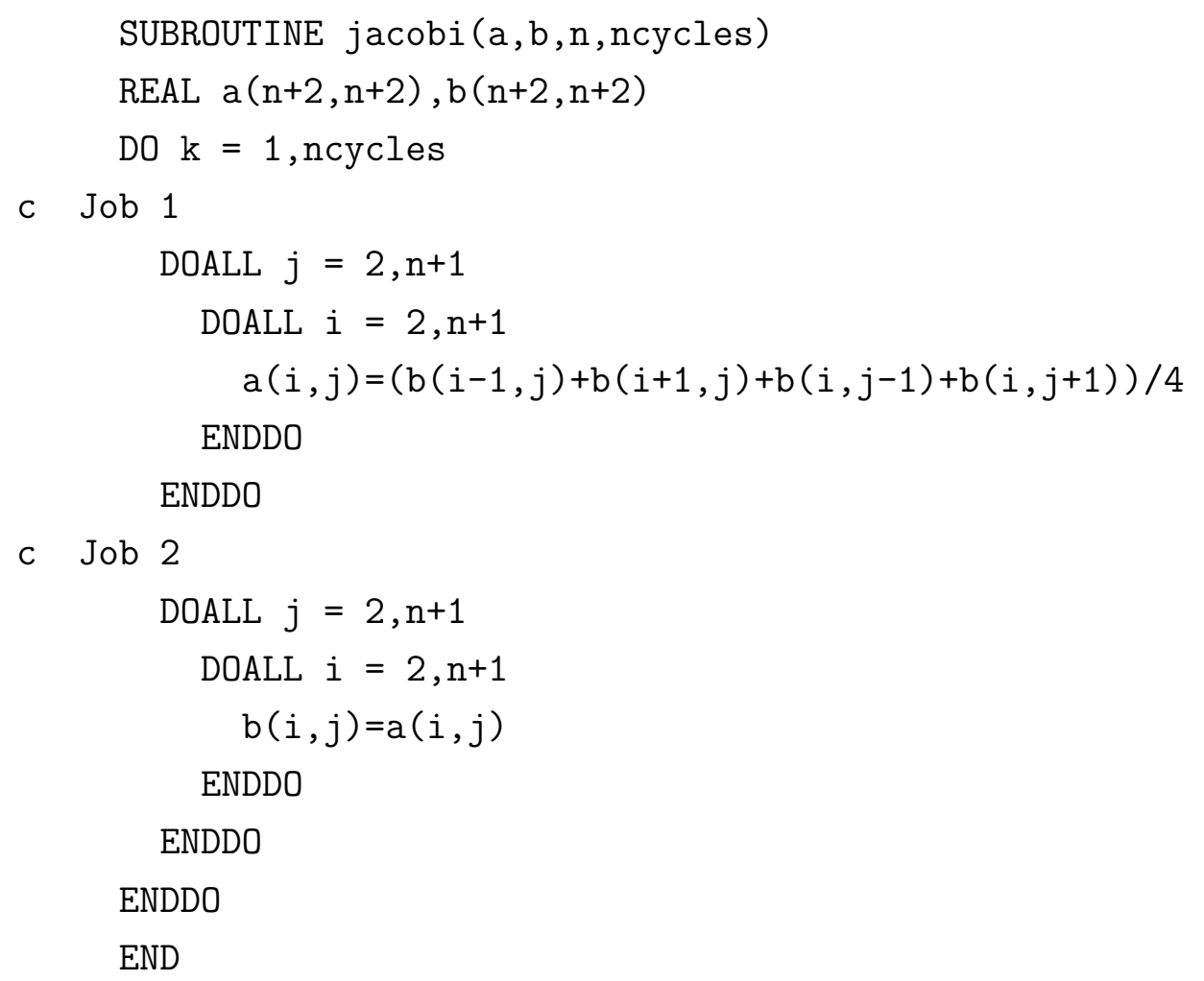

The outer DOALL-loops are partitioned as Job 1 and 2. The original program is 19 lines, the resulting PVM-codes for master and slave contain respectively 107 and 91 lines. If one roughly assumes that the programming time is proportional to the number of lines in a program, the productivity gained by the automatic PVM-code generation and data distribution is about a factor of ten. With respect to data compaction, the areas of matrix b in Job 1 are $B_{1}=(1: n: 1,2: n+1: 1), B_{2}=(3: n+2: 1,2: n+1: 1)$, $B_{3}=(2: n+1: 1,1: n: 1)$ and $B_{4}=(2: n+1: 1,3: n+2: 1)$. Since the strides in both dimensions $\mathrm{i}$ and $\mathrm{j}$ are $s_{i}=s_{j}=1$, areas $B_{1}, B_{2}, B_{3}$ and $B_{4}$ fully overlap in the shared region $R=(3: n: 1,3: n: 1)$. Consequently, the PVM generator sends the 
data in region $R$ once, resulting in a data reduction of 4 . After merging region $R$ with the boundary rows and columns, matrix $\mathrm{b}$ is packed into the message buffer info by the following code:

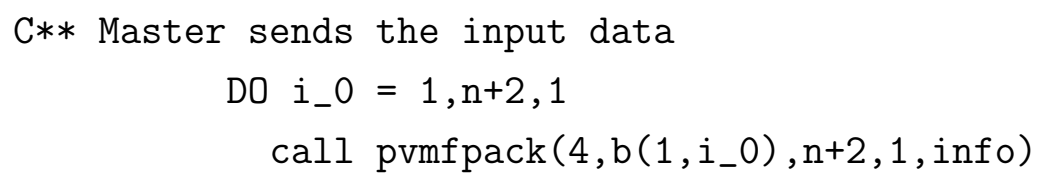

\section{Conclusion}

FPT contains many of the dependence tests described in Banerjee's comprehensive work [8] and in addition includes the analysis of IF statements by sharpening the iteration space and constant propagation. Backward GOTO's are converted into DOWHILE loops and these are furthermore changed into DO-loops when the while condition can be expressed by a linear induction variable[33].

The treatment of loop transformations in the interactive mode is inspired by the wellknown tiny-tool developed by Wolfe [30]. The user can select and clip any particular loop or nested loop, edit, modify and apply the loop transformations such as interchange, skewing, strip mining, wavefront as well as unimodular transformations. In each step the user can perform a dependence test and verify the parallelism gained by the transformation.

At the end of the interactive phase, an annotated pretty printed source code is generated, instrumented with parallel directives for several platforms. These include a shared memory Solaris machine, a parallel intermediate language for a multiprocessor prototype (VPS) as well as threaded code and PVM.

FPT-PVM facilitates high performance computing in a distributed network of computers by translating a sequential application into parallel PVM code. The resulting program uses only standard f77 and PVM library calls. Furthermore, the program is highly readable and informative to the user. Finally, the application of the FPT-PVM system is straightforward and has been demonstrated to allow efficient distributed computing. 


\section{References}

[1] Aho, A. V., and Ullman, J. D., Compilers: Principles, Techniques, and tools. AddisonWesley, Reading, MA, 1986.

[2] Allen, J.R., "Automated Environments for Parallel Programming", IEEE Software February, Vol 7, pp. 21-29, 1985.

[3] Allen, J.R., "Dependence Analysis for subscript Variables and Its Application to Program Transformations", Ph.D. Dissertation, Department of Mathematical Sciences, Rice University, Houston, Texas(April), 1983.

[4] Allen, J.R., Kennedy, K., Porterfield, C., and Warren, J., "Conversion of Control Dependence to Data Dependence", in Conference Proceedings - The 10th Annual ACM Symposium on Principles of Programming Languages, 1983, pp. 177-189.

[5] Allen, J.R., and Kennedy, K., "Automatic Translation of FORTRAN Programs to Vector Form". ACM Transaction on programming Language\& Systems. Vol. 9, No. 4(October), pp. 491-542, 1984.

[6] Ammarguellat Zahira, A Control-Flow Normalization Algorithm and its Complexity, IEEE Transactions on Software Engineering, Vol. 18, 3, pp. 237-251, 1992

[7] Baker, B., "An algorithm for structuring flowgraphs," JACM, vol 24, no. 1, 1977.

[8] Banerjee U., Loop Transformations for Restructuring Compilers, Kluwer Academic Publishers, Boston (Mass.), 305 pp., 1993.

[9] Banerjee U., Eigenmann R., Nicolau A., Padua D., Automatic Program Parallelization, IEEE Proceedings, Vol. 81, 2, pp. 211-243, 1993.

[10] Banerjee, U., Dependence Analysis for Supercomputing, The Kluwer international series in engineering and computer science. Parallel processing and fifth generation computing. ISBN 0-89838-289-0. Kluwer Academic Publishers, 1988.

[11] Brandes, T., ADAPTOR Programmer's Guide (Version 3.1) Technical documentation, GMD, Oct., 1995. Available via anonymous ftp from ftp.gmd.de as gmd/adaptor/docs/pguide.ps.

[12] Chang S-L., Du D.H-C., Hsieh J., Tsang R.P., Lin M., Enhanced PVM Communications over a High-Speed LAN, IEEE Parallel \& Distributed Technology, Fall '95, pp. 20-32, 1995. 
[13] D'Hollander E.H., Partitioning and Labeling of Loops by Unimodular Transformations, IEEE Transactions on Parallel and Distributed Systems, Vol. 3, 7, pp. 465-476, 1992.

[14] D'Hollander E.H., The VPS, a Virtual MIMD Processor and its Software Environment, Workshop on Compiling Techniques and Compiler Construction, for Parallel Computers, Oxford UK, 13-15 September 1989, 19-36, 1989.

[15] Dongarra J., Geist G.A., Manchek R., Sunderam V.S., Integrated PVM framework supports heterogeneous network, Tennessee Univ., Knoxville, TN, USA Computers in Physics Vol: 7 Iss:2 pp. 166-74, 1993.

[16] Dunigan T.H., Hall K.A., PVM and IP Multicast, Oak Ridge National Laboratory, Technical Report ORNL/TM-13030, 28 pp., 1996.

[17] Ferrante, J., Ottenstein, K. J., and Warren, J. D., "The Program Dependence Graph and Its use Optimization", ACM Trans. on Programming Languages and Systems. vol. 9 no. 3, July, pp. 319-349, 1987.

[18] Geist A., Beguelin A., Dongarra J., Jiang W., Manchek R., Sunder,PVM 3 User's Guide and Reference Manual, Oak Ridge National Laboratory, Oak Ridge TN 37831-6367 Technical Report, ORNL/TM-12187, May, 108pp., 1993.

[19] Gupta M., Banerjee P., Demonstration of Automatic Data Partitioning Techniques for Parallelizing Compilers on Multicomputers, IEEE Trans. on Parallel Distributed System. Vol. 3, No. 2, March, pp. 179-93, 1992.

[20] Kuck, D.J., Kuhn, R.H., and Padua, D.A., Leasure, B.R., and Wolfe, M.J. "Dependence Graphs and Compiler Optimizations", Conference Proceedings- The 8th Annual ACM Symposium on Principles of Programming Languages (Williamsburgh, Virginia, January 26-28), ACM Press, pp. 207-218, 1981.

[21] Lundstrom Stephen F. and Barnes George H., "A Controllable MIMD Architecture", International Conference on Parallel Processing, pp. 19-27, 1980.

[22] Polychronopoulos, C., Girkar, M., Haghighat, M.R., Lee, C.H., Leungg, "Parafrase-2: An Environment for Parallelizing, Partitioning, Synchronizing and Scheduling Programs on Multiprocessors", CSRD Urbana-Champaign IL 61801 USA, ICPP'89, II - Software, pp. 39-48, 1989.

[23] Ramshaw, L., "Eliminating go to's while Preserving Program Structure", JACM. Vol. 35, No. 4, October, pp. 893-920, 1988. 
[24] Sivaraman, H., Raghavendra, C.S., Parallelizing sequential programs to a cluster of workstations, Proceedings of the 1995 Workshop on Challenges for Parallel Processing, Raleigh, NC, USA. 14 Aug., pp. 38-41, 1995.

[25] Sunderam V.S., Geist G.A., Dongarra J., Manchek R., The PVM Concurrent Computing System: Evolution, Experiences, and Trends, Parallel Computing, 1992.

[26] Tarjan, R. E., "Testing Flow Graph Reducibility", Computer Journal. vol. 9, pp. 355$365,1974$.

[27] Williams, M. H., "Generating structured flow diagrams: the nature of unstructuredness", Computer Journal. vol 20, no. 1, pp. 45-50, 1977.

[28] Williams M. H, and Ossher, H. L., "Conversion of Unstructured flow diagrams to structured form", Computer Journal. vol. 21, no. 2, pp. 161-167, 1978.

[29] Wolfe, M.J., "Optimizing Supercompilers for Supercomputers", PhD. Thesis, the Graduate College of the University of Illinois at Urbana-Champaign, 1982.

[30] Wolfe M., The Tiny Loop Restructuring Research Tool, Proceedings of the International Conference on Parallel Processing, vol. II - Software, pp. 46-53, 1991.

[31] Wolfe, M., "Beyond Induction Variables", ACM SIGPLAN'92, SIGPLAN Notices. Vol. 27, No. 7 (July), pp. 162-174, 1992.

[32] Wu, Y., Lewis, T.G., "Parallelizing While Loops", Proceedings of the International Conference on Parallel Processing '90, II - Software, August 13-17, pp. 1-8, 1990.

[33] Zhang Fubo, D'Hollander E.H., Extracting the Parallelism in Programs with Unstructured Control Statements, International Conference on Parallel and Distributed Systems, ICPADS'94, Taipei, December, 1994.

[34] Zhang Fubo, FPT: A Parallel Programming Environment, PhD. Thesis, September, 26, pp. 203, 1996.

[35] Zhu, C.Q., and Yew, P.C., "A Scheme to Enforce Data Dependence on Large Multiprocessor System", IEEE Trans. on Software and Engineering, 13, 6, pp. 352-358, 1987. 